\title{
Evolution après incendie de la structure de quelques phytocénoses méditerranéennes du Bas-Languedoc (Sud de la France)
}

\author{
L. TRABAUD \\ Département d'Ecologie générale, \\ Centre d'Etudes phytosociologiques et écologiques L. Emberger, C.N.R.S. \\ route de Mende, B.P. 5051, F 34033 Montpellier Cedex
}

\section{Résumé}

L'évolution de la structure de la végétation des garrigues après incendie a été étudiée sur 47 placettes réparties dans le Bas-Languedoc. C'est la méthode directe (ou diachronique) qui a été utilisée pendant la douzaine d'années qu'ont été suivies les placettes incendiées. Huit types de communautés végétales représentatives de la région ont été étudiés, allant des taillis de chêne vert aux pelouses de brachypodes.

Après le feu, l'accroissement quantitatif de la végétation tend à retourner vers une structure comparable à celle qui existait avant l'incendie. Assez rapidement, la végétation réapparaît et recouvre la surface du sol. L'accroissement vertical a été aussi considéré : au fur et à mesure que les communautés vieillissent, l'importance des strates basses $(<25 \mathrm{~cm})$ diminue, tandis que celle des strates hautes augmente.

\section{Introduction}

Du fait de sa fréquence passée et présente, le feu est un facteur influençant la dynamique des communautés végétales de la région méditerranéenne française. Divers auteurs (Braun-Blanquet, 1936 ; Kunholtz-Lordat, 1938, 1958 ; KorNas, 1958; BARRY, 1960) ont considéré l'évolution de la végétation sous la forme d'une succession de stades dans les séries régressives. Cependant, cette proposition est faite à partir d'observations effectuées sans que le devenir de la végétation après le traumatisme créé par le feu ait été réellement suivi.

Pour savoir quel est le devenir réel des phytocénoses après le feu, il est essentiel de suivre leur évolution au cours du temps dans la nature sur des placettes qui ont subi des feux sauvages dont la date exacte du dernier feu est bien connue. Quels sont, alors, les éléments des problèmes rencontrés lors d'une étude concernant les communautés végétales de la zone des garrigues du Bas-Languedoc brûlées par des feux sauvages?

Le passage de la flamme élimine toute la végétation qui se trouve à la surface et au-dessus de la surface du sol. Il est intéressant d'étudier quels sont les végétaux qui sc réinstallent après le feu et quelles communautés ils vont constituer. L'évolution pourrait avoir lieu selon quatre directions : 
1) une ouverture plus importante de ce qu'était le couvert végétal avant le feu, associée à une diminution de la proportion des végétaux ligneux ;

2) un retour à la végétation antérieure, après un laps de temps plus ou moins long et des stades intermédiaires ;

3) un retour immédiat à la végétation antérieure, sans stade de transition ;

4) une évolution progressive vers des communautés différentes de celle qui a brûlé mais plus proches de celles en équilibre avec les conditions du climat.

Or, l'étude diachronique de l'évolution de la végétation et de la flore après des feux sauvages dans le Languedoc montre que le retour à un état proche de l'état initial (celui qui préexistait au feu) est rapide (Trabaud, 1980; Trabaud \& Lepart, 1980). Par ailleurs, des résultats similaires ont été trouvés par des auteurs travaillant sur des communautés végétales analogues (chaparral californien : Sampson, 1944; Horton \& Kraebel, 1955 ; SweEney, 1956, 1967 ; Hanes, 1971 ; maquis israélien : NaveH, 1974, 1975; fynbos sud-africain : KRUGER, 1977 ; scrub australien : SPECHT et al., 1958 ; Purdie \& Slatyer, 1976).

Au fur et à mesure que les plantes vont occuper le territoire brûlé, un agencement plus complexe de l'architecture des communautés va se produire entrainant des changements : les végétaux vont occuper l'espace à la fois horizontalement et verticalement. Comment évoluera ce développement ? Les changements seront-ils comparables entre les différentes communautés? Autrement dit, le blocage de l'évolution floristique provoquera-t-il un blocage de l'évolution des phytocénoses, ou vice versa ? Y a-t-il un retour vers une structure comparable à celle qui existait avant le feu ?

Pour tenter de répondre à ces questions, la reconstitution de zones brûlées a été étudiée à la fois sous l'angle floristique et structural. Seuls, les changements de la structure sont considérés dans ce travail.

\section{Méthode, dispositif et nature des observations}

Pour suivre les étapes de la reconstitution de la végétation dans une zone brûlée, nous avons choisi la méthode directe (PAviLlard, 1935), ou méthode «diachronique». Il s'agit de suivre au cours du temps les modifications de la végétation sur des placettes permanentes installées après le passage du feu. Cette méthode, assez contraignante, permet de mettre en évidence des variations à la fois floristiques et stucturales relativement faibles.

L'étude a porté principalement sur les premières années qui suivent l'incendie (une dizaine d'années); elles sont capitales, car c'est pendant cette période que la reconstitution de la végétation est la plus spectaculaire.

Les 47 placettes d'observation retenues sont situées dans la région du Bas-Languedoc, installées dans des communautés végétales ayant brûlé et représentatives des communautés les plus fréquemment rencontrées dans cette région. Leur étude floristique a permis de les rattacher à des associations bien déterminées (Trabaud, 1980). Elles représentent : 
- des taillis denses de chêne vert (Quercus ilex L.) (1), lorsque le recouvrement du chêne vert a dépassé 50 p. 100 trois ans après le feu : 7 stations; rattachés floristiquement au Quercetum ilicis ;

- des taillis clairs de chêne vert (Quercus ilex L.), lorsque le recouvrement du chêne vert a toujours été inférieur à 50 p. 100 pendant les années d'observation : 6 stations ; rattachés au Quercetum ilicis;

- des garrigues denses de chêne kermès (Quercus coccifera L.), lorsque le recouvrement du chêne kermès a dépassé 90 p. 100 trois ans après le feu : 10 stations; rattachées au Cocciferetum;

- des garrigues claires de chêne kermès (Quercus coccifera L.), lorsque le recouvrement du chêne kermès a toujours été inférieur à 90 p. 100 : 5 stations ; rattachées at: Cocciferetum;

- des pinèdes de pin d'Alep (Pinus halepensis Mill.) : 7 stations ; rattachées au Rosmarineto-Lithospermetum pinetosum;

- des garrigues de romarin (Rosmarinus officinalis L.) : 6 stations ; rattachées au Rosmarineto-Lithospermetum ;

- des pelouses de brachypode rameux (Brachypodium retusum (Pers.) Beauv.) : 3 stations; rattachées au Brachypodietum ramosi;

- des pelouses de brachypode phoenicoïde (Brachypodium phoenicoides (L.) R. et S.) : 3 stations; rattachées au Brachypodietum phoenicoidis.

Il a été possible de localiser et de choisir les emplacements des placettes étudiées grâce aux informations recueillies lors de l'exploitation des rapports d'incendies établis depuis 1962. Ce travail a permis de dresser une carte des zones incendiées dans le département de l'Hérault (Trabaud, 1980). La date d'incendie de chaque zone étudiée est donc parfaitement connue : ce sont toujours des feux d'été (période de juin à août inclus). Après reconnaissance sur le terrain des zones brûlées, le critère du choix des stations d'étude était déterminé par l'homogénéité apparente des différents types de peuplements.

Pour suivre l'évolution de la structure, la technique d'observation consiste en une ligne permanente (Levy \& Madden, 1933; Long, 1957, 1958 ; Daget \& Poissonet, 1971) de $20 \mathrm{~m}$ de long. Les piquets repères sont scellés dans le sol. Les lectures sont faites tous les $10 \mathrm{~cm}$. A chaque série d'observations, la présence au point, ainsi que le nombre de contacts par taxon et par strate, sont notés. Ces deux types de mesures permettent d'obtenir une estimation de l'occupation de l'espace par la végétation.

Les observations ont été faites régulièrement tous les ans au printemps pendant les cinq premières années qui ont suivi le feu. Par la suite, au fur et à mesure que les peuplements donnaient l'impression de se stabiliser physionomiquement, les observations n'étaient plus effectuées que tous les deux ans.

\section{Résultats : accroissement quantitatif de la végétation}

Le passage du feu influence non seulement la composition floristique des communautés, mais il modifie aussi l'agencement des végétaux et leur phytomasse. L'agencement correspond à la répartition spatiale, aussi bien sur le plan vertical que sur le plan horizontal, des différentes plantes qui constituent les communautés.

(1) La nomenclature taxinomique est celle de Flora Europaea (Tutis et al., 1964-1980). 


\subsection{Accroissement horizontal}

Assez rapidement après un incendie, la végétation réapparaît et recouvre la surface du sol. En effet, généralement, quinze jours à un mois après le feu, commencent à apparaître les premiers rejets; puis progressivement la végétation devient de plus en plus dense.

$\mathrm{Si}$, lors des observations le long de la ligne de mesure, la présence d'un taxon au point de lecture est seulement prise en compte, le nombre de points sous lesquels un taxon est observé donne la "fréquence spécifique FS (GoDron, 1968; DAGET \& Poissonet, 1971, 1974). Si le nombre d'observations de la présence d'un taxon est ramené au pourcentage du nombre total de points observés par ligne, on obtient la «fréquence centésimale» $F C$ du taxon qui est une estimation au sens exact du mot, du recouvrement de ce taxon (GODRON, 1968). On peut alors considérer que la fréquence centésimale du taxon représente son recouvrement. Comme les taxons se superposent les uns aux autres, la fréquence centésimale totale peut être supérieurc à 100 p. 100.

Les communautés étudiées présentent deux types daccroissement horizontal selon qu'elles sont dominées soit par des végétaux ligneux, soit par des végétaux herbacés.

Comme la végétation se réinstalle rapidement après le passage de la flamme, son recouvrement total atteint très vite 80 p. 100 de la surface du sol (un an après le feu pour les taillis denses de chêne vert et les garrigues denses de chêne kermès, par exemple), il semble préférable de suivre lévolution du recouvrement en tenant compte des principales formes biologiques (RAUNKIAER, 1905; Godron et al., 1968), ou de groupes de formes, des végétaux qui composent les communautés. Pour ce faire, les végétaux ont été réunis en fonction de leurs formes potentielles, cest-à-dire les formes qu'ils atteindraient normalement en absence de feu ou de tout autre traumatisme. Ainsi, ont été constitués quatre grands groupes (2):

- les végétaux ligneux qui peuvent dépasser $25-30 \mathrm{~cm}$ de hautcur, soit des phanérophytes ligneux;

- ceux qui correspondent aux végétaux ligneux ne dépassant jamais, ou rarement, $25-30 \mathrm{~cm}$ de hauteur, soit des chaméphytes ligneux; herbacés ;

- les graminées qui forment un groupe bien identifiable parmi les végétaux

- et les végétatix herbacés antres que les graminées.

\subsection{Communautés dominées par des végétaux ligneux}

Dans les communautés où les végétaux dominants, avant le feu, sont des ligneux hauts, c'est-à-dire supérieurs à $2 \mathrm{~m}$ (Godron et al., 1968): taillis de chêne vert, pinèdes, le recouvrement des phanérophytes ligneux s'accroît régulièrement au fur et à mesure que les communautés avancent en âge (fig. 1). Probablement, au-delà de la durée des observations (12 ans après le feu), un ralentissement devrait commencer à se faire sentir, les communautés atteignant un stade d’équilibre proche du stade adulte.

(2) Un tableau annexe donne la liste des principaux taxons les plus fréquemment rencontrés et leur présence dans les communautés étudiées. 


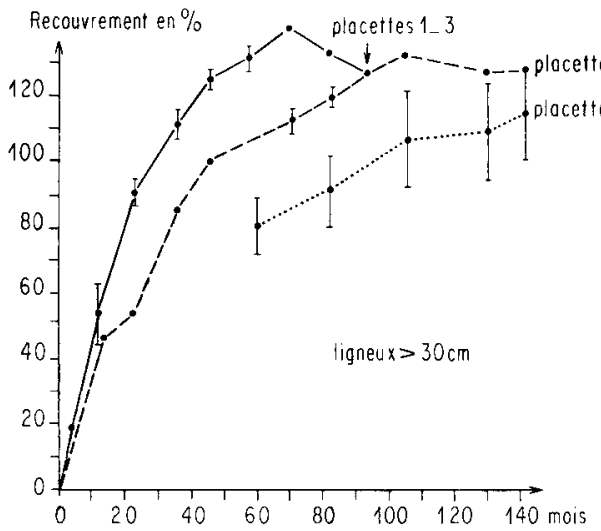

a. Toillis denses de

Chêne vert

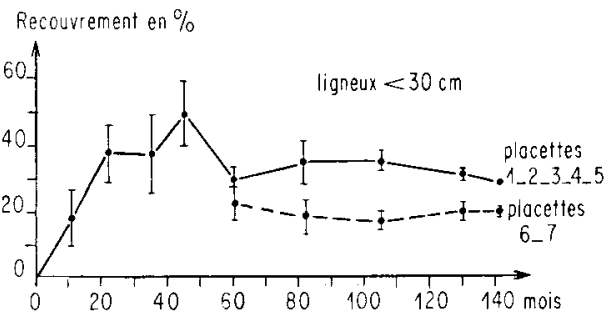

Recouvrement en \%

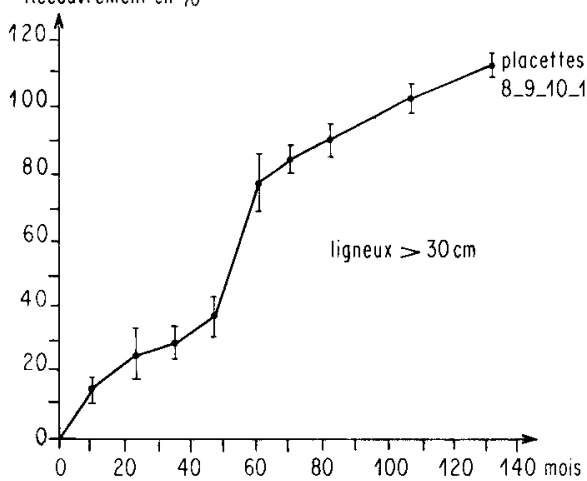

Recouvrement en \%
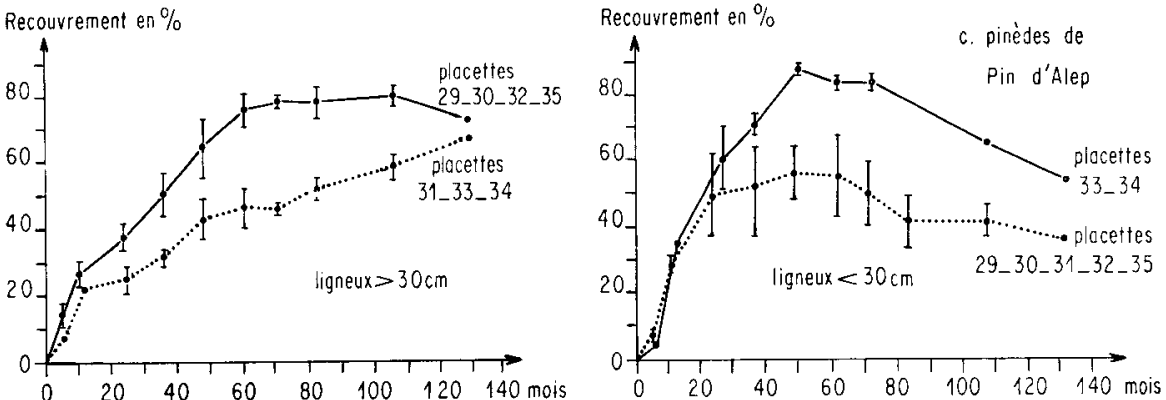

FIG. 1

Evolution, au cours du temps, du recouvrement des végétaux ligneux dans les communautés ligneuses hautes incendiées.

Cover changes through time of woody plants in burnt communities initially dominated by trees $(>2 \mathrm{~m})$. 
Le recouvrement des chaméphytes ligneux passe par un maximum généralement situé vers la quatrième année (entre 40 et 60 mois après l'incendie), puis décroît légèrement, sauf pour les taillis clairs de chêne vert où le recouvrement des chaméphytes ligneux tend à rester constant tout au long des années d'observations, même lorsque les peuplements atteignent dix ans et plus.
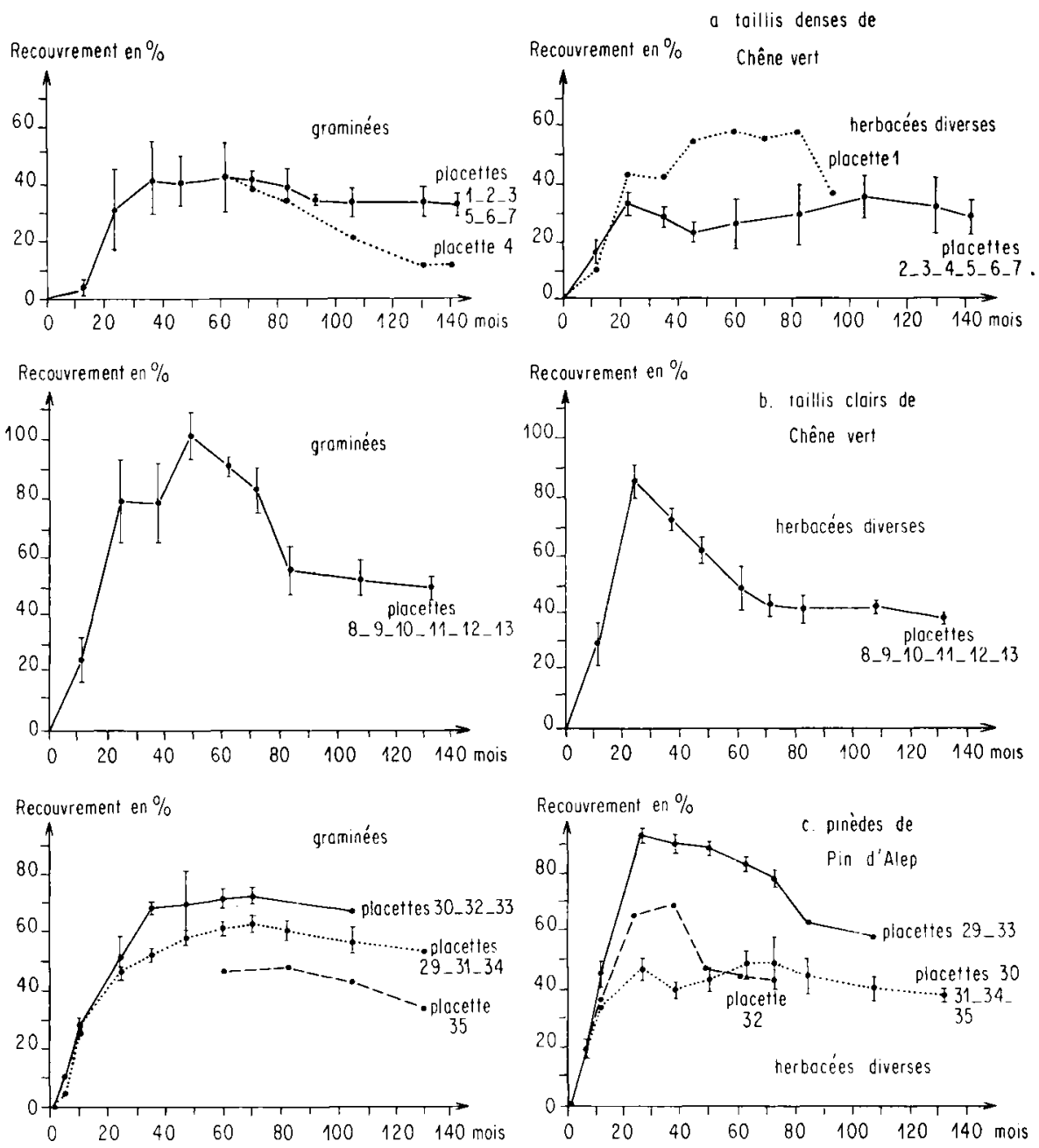

Fig. 2

Evolution, au cours du temps, du recouvrement des végétaux herbacés dans les communautés ligneuses hautes incendiées.

Cover changes through time of herbaceous plants' in burnt communities initially dominated by trees $(>2 \mathrm{~m})$.

L'évolution du recouvrement des graminées (fig. 2) s'apparente à celle déjà décrite pour les chaméphytes ligneux. Ce recouvrement passe par un maximum situé, ici aussi, 
à différentes époques après le feu, mais toujours au cours des cinq premières années qui suivent l'incendie. Par la suite, le recouvrement total des graminées diminue de moins en moins rapidement au fur et à mesure que les communautés avancent en âge, pour se stabiliser au niveau qui a été mesuré dans les stades les plus adultes étudiés.

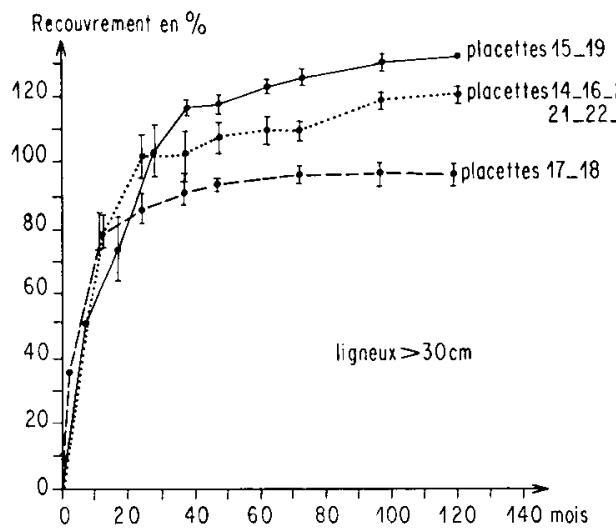

0. garrigues denses de

Chêne kermès

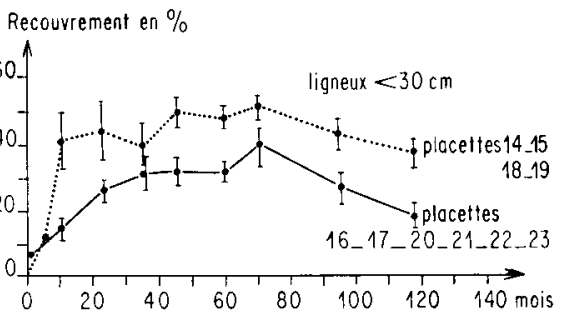

Recouvrement en $\%$

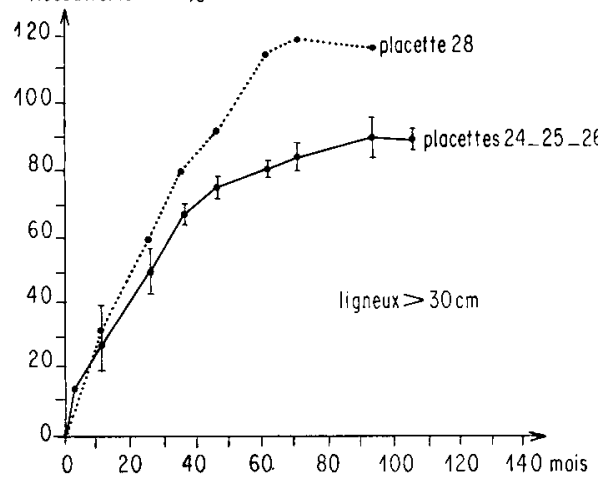

b. garrigues claires de

Chêne kermès

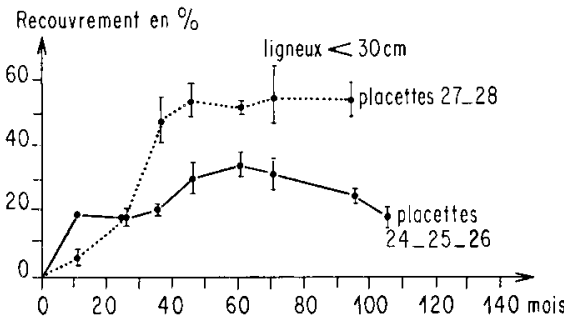

Recouvrement en $\%$

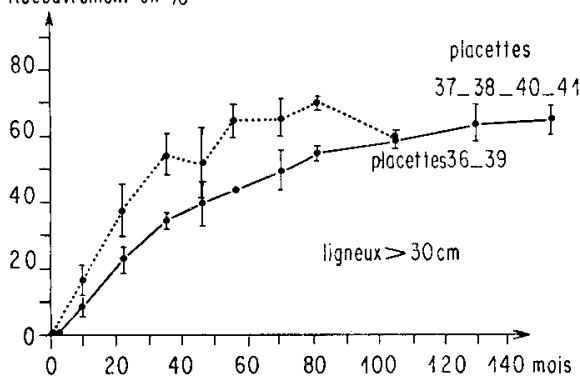

Recouvrement en $\%$

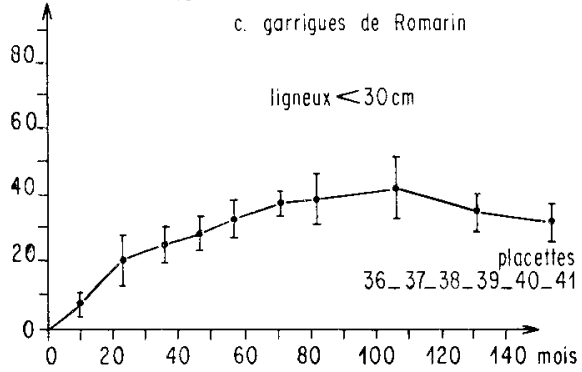

FIG. 3

Evolution, au cours du temps, du recouvrement des végétaux ligneux dans les communautés ligneuses basses incendiées.

Cover changes through time of woody plants in burnt communities initially dominated by shrubs $(<2 \mathrm{~m})$. 
Pour les «végétaux herbacés autres que les graminées », quelle que soit la communauté, le recouvrement atteint son point maximal vers la deuxième année, puis diminue (fig. 2) pour tendre à se stabiliser.

Dans le cas des formations à dominance de ligneux bas, c'est-à-dire dont la hauteur ne dépasse pas. $2 \mathrm{~m}$ (garrigues de chêne kermès, garrigues de romarin), le recouvrement des phanérophytes ligneux, après un accroissement assez rapide au cours des cinq premières années après l'incendie, particulièrement pour les garrigues de chêne kermès, tend à rester constant au fur et à mesure que ces communautés avancent en âge (au-delà de 60 mois après le feu, figure 3 ).
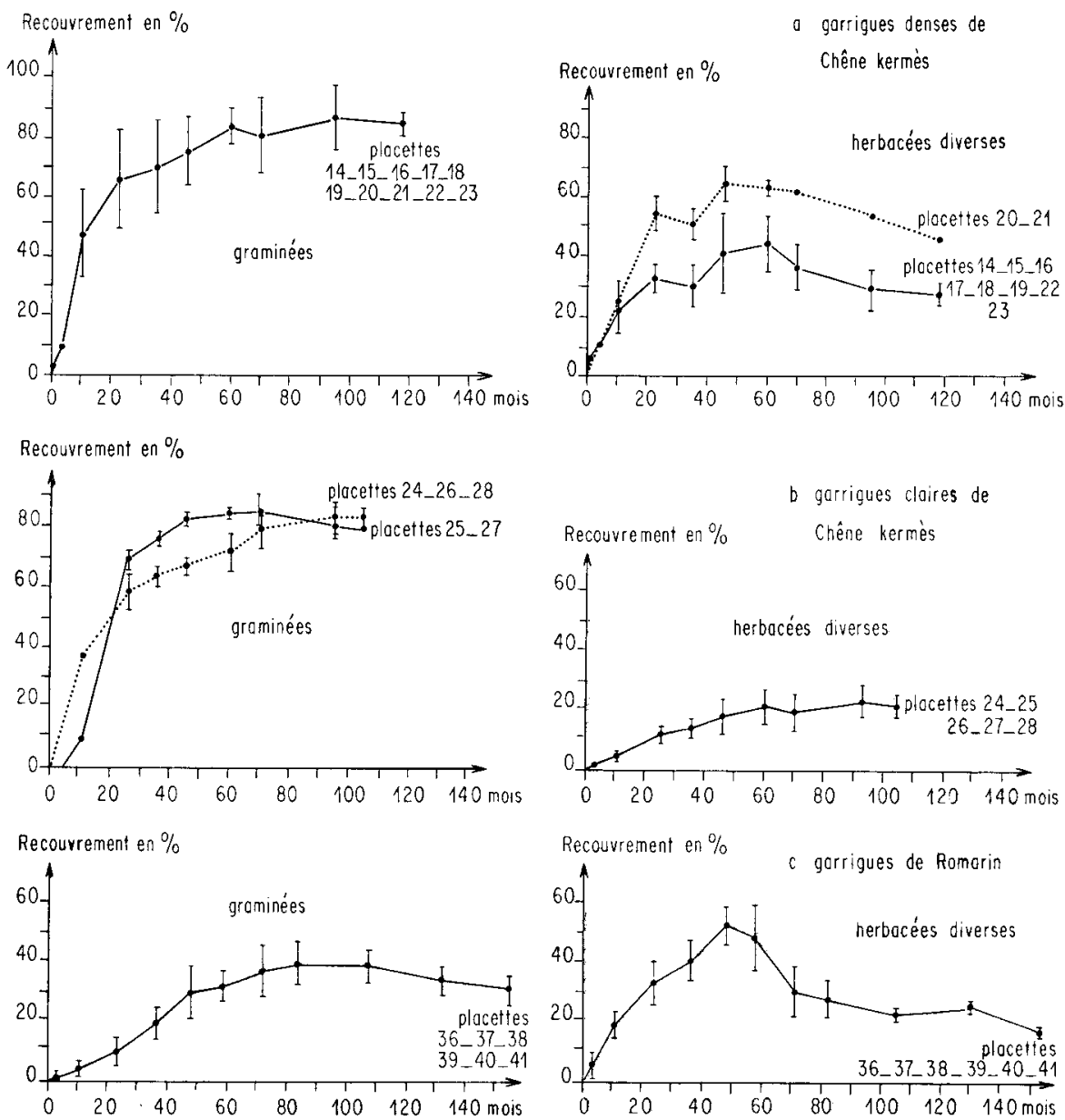

FIG. 4

Evolution, au cours du temps, du recouvrement des végétaux herbacés dans les communautés ligneuses basses incendiées.

Cover changes through time of herbaceous plants in burnt communities initially dominated by shrubs $(<2 \mathrm{~m})$. 
Selon les communautés, le recouvrement des chaméphytes ligneux atteint un maximum à des moments différents, qui peuvent être situés entre 2 et 9 ans, puis il diminue progressivement.

A l'inverse du phénomène observé dans les communautés de ligneux hauts, le recouvrement de l'ensemble des graminées, après avoir atteint son maximum, ne décroît pas (fig. 4). Il tend à rester constant au fur et à mesure que les communautés «vieillissent », tout au moins pendant la durée des observations (10 à 12 ans).

Sauf pour les garrigues claires de chêne kermès, le recouvrement des « herbacées autres que les graminées » passe par un maximum vers la cinquième année puis diminue progressivement.

\subsection{Communautés dominées par des végétaux herbacés}

Dans les communautés dominées par des végétaux herbacés (ce sont les pelouses de brachypode rameux et celles de brachypode phoenicoïde), l'évolution du recouvrement des diverses formes biologiques est totalement différente de celle décrite précédemment pour les communautés dominées par les végétaux ligneux.

Les phanérophytes ligneux ne jouent plus qu'un rôle négligeable et leur recouvrement est très faible au cours des années d'observation (fig. 5). Cependant, celui-ci présenterait une légère tendance à un accroissement. Ce qui peut faire penser à un envahissement progressif par des végétaux ligneux, suggérant l'initiation d'une succession conduisant vers d'autres communautés. Cela est tout à fait vraisemblable dans le cas d'une évolution normale, en absence de tout traumatisme, de la végétation vers un stade climacique ou paraclimacique.

Le recouvrement total des chaméphytes ligneux n'est pas identique dans les deux types de pelouses. Dans les pelouses de brachypode rameux, passé le temps d'installation après le feu, les chaméphytes ligneux conservent un recouvrement constant au cours des années d'observation (fig. 5 a). En revanche, dans les pelouses de brachypode phoenicoïde, au cours de la période comprise entre la deuxième et la cinquième année qui suit le feu, les chaméphytes ligneux présentent un accroissement important, qui décroît brutalement après la cinquième année (fig. 5 b). Ce phénomène, constaté pour les trois placettes étudiées, bien que les feux n'aient pas eu lieu la même année, pourrait être expliqué par un accroissement du recouvrement des graminées (fig. $6 \mathrm{~b}$ ) et donc par un phénomène de compétition pour les éléments nutritifs ou pour la lumière : Brachypodium phoenicoides, formant des touffes denses, serrées et relativement hautes $(50 \mathrm{~cm})$, tendant à dépasser en hauteur les chaméphytes ligneux, aurait un pouvoir étiolant.

Dans toutes les communautés dominées par les végétaux ligneux étudiées jusquà présent, les plantes annuelles (thérophytes) ne jouent qu'un rôle négligeable, si ce n'est nul, dans l'accroissement quantitatif de la phytomasse. Au contraire, dans les pelouses, les théophytes sont présents en très grand nombre et participent à la structure des communautés. Il est donc préférable de distinguer les «thérophytes » des autres végétaux herbacés.

Le recouvrement des «graminées» devient de plus en plus important au fur et à mesure que les communautés avancent en âge. Les "végétaux herbacés autres que les 
graminées > présentent un recouvrement élevé au cours des cinq premières années qui suivent un feu; par la suite, ce recouvrement se stabilise (fig. 6).

En revanche, le recouvrement des «thérophytes » est très variable ; cela est dû à la nature des communautés elles-mêmes et à l'époque des relevés, mais aussi, probablement, aux conditions climatiques qui précèdent les relevés (BHarucha, 1932; Soroceanu, 1936).

\subsection{Accroissement vertical}

Le nombre de «contacts» faits par une plante le long de la génératrice d'une aiguille de visée placée perpendiculairement au sol donne une approximation de la quantité de matériel végétal épigé (P. \& J. Poissonet, 1969; Daget \& Poissonet, 1971, 1974). Il permet d'appréhender la répartition de l'accroissement vertical de la végétation en fonction de la stratification et de la hauteur.

a. pelouses de Brachypode romeux
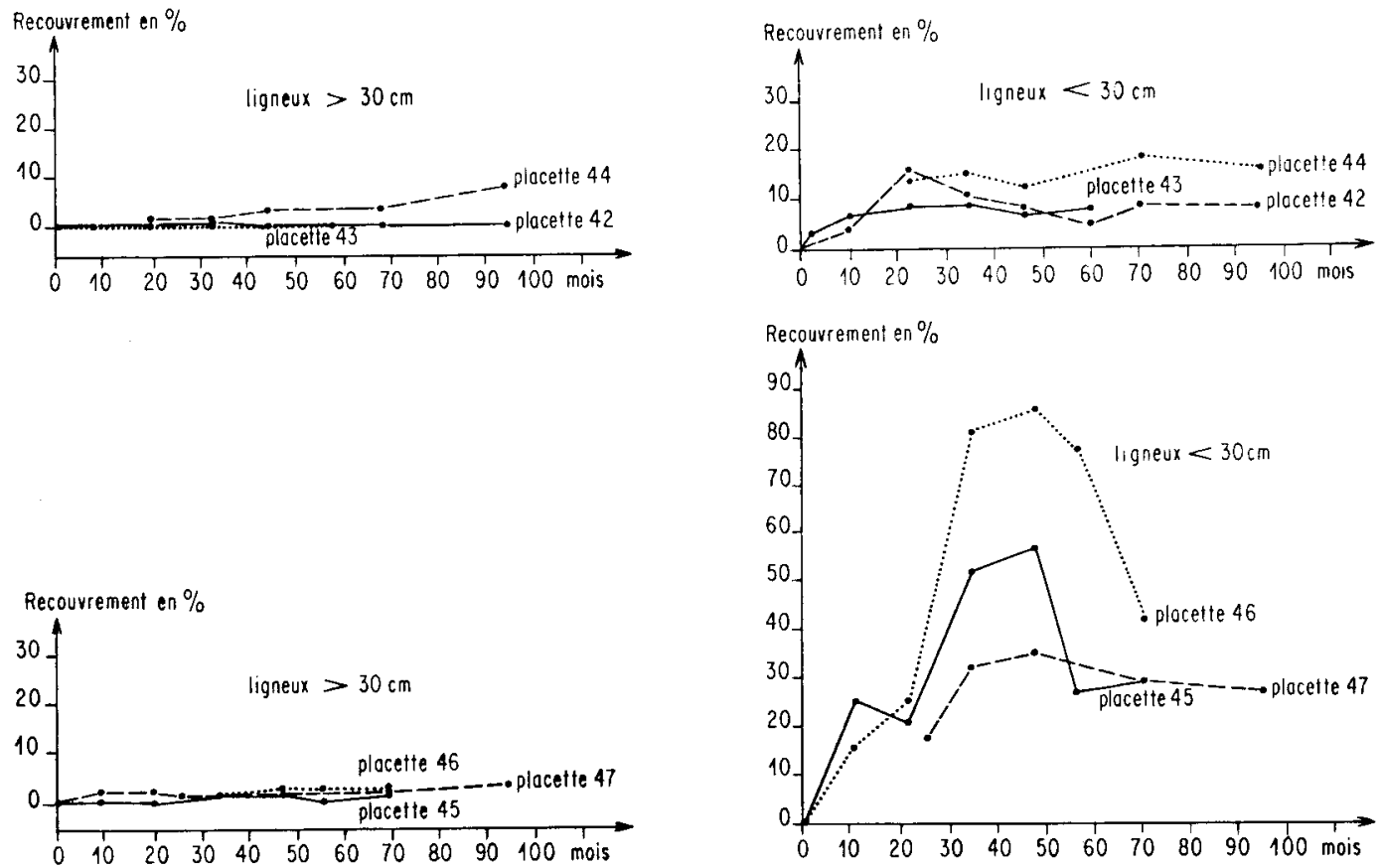

b. pelouses de Brochypode phænicoïde

FIG. 5

Evolution, au cours du temps, du recouvrement des végétaux ligneux dans les communautés herbacées incendiées.

Cover changes through time of woody plants in burnt community initially dominated by herbaceous plants. 

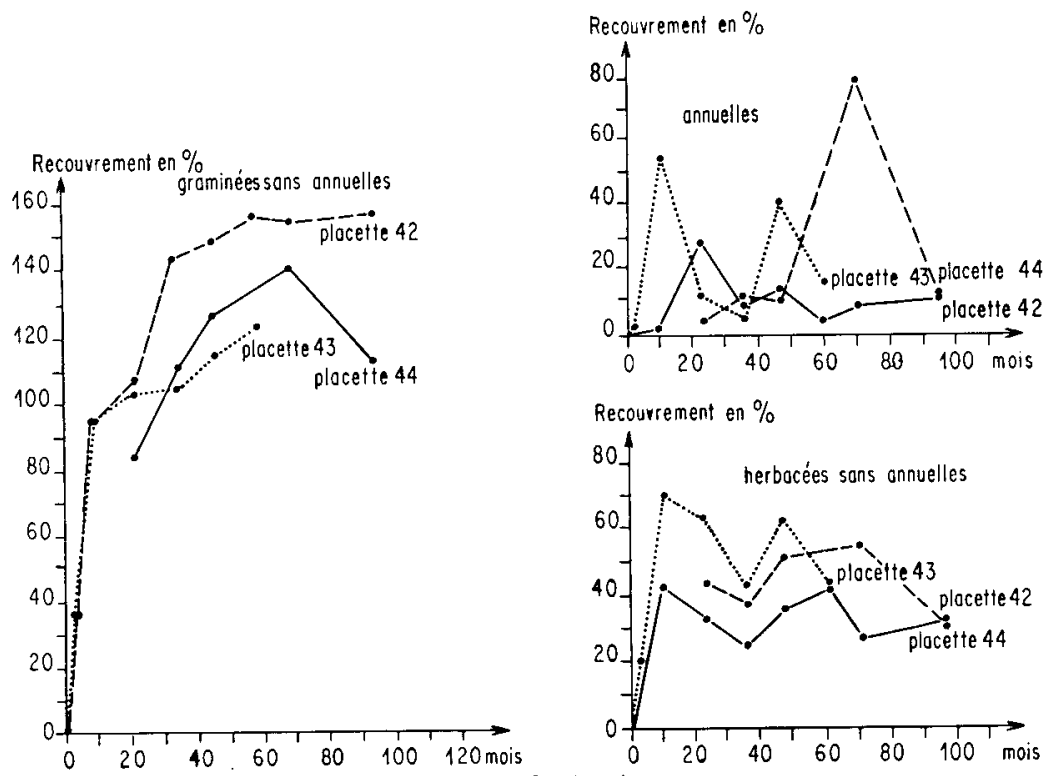

Recourrement en $\%$

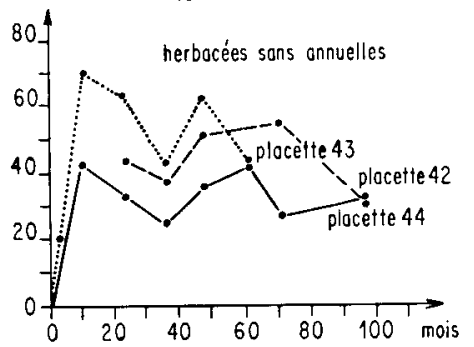

a. pelouses de Brachypode romeux

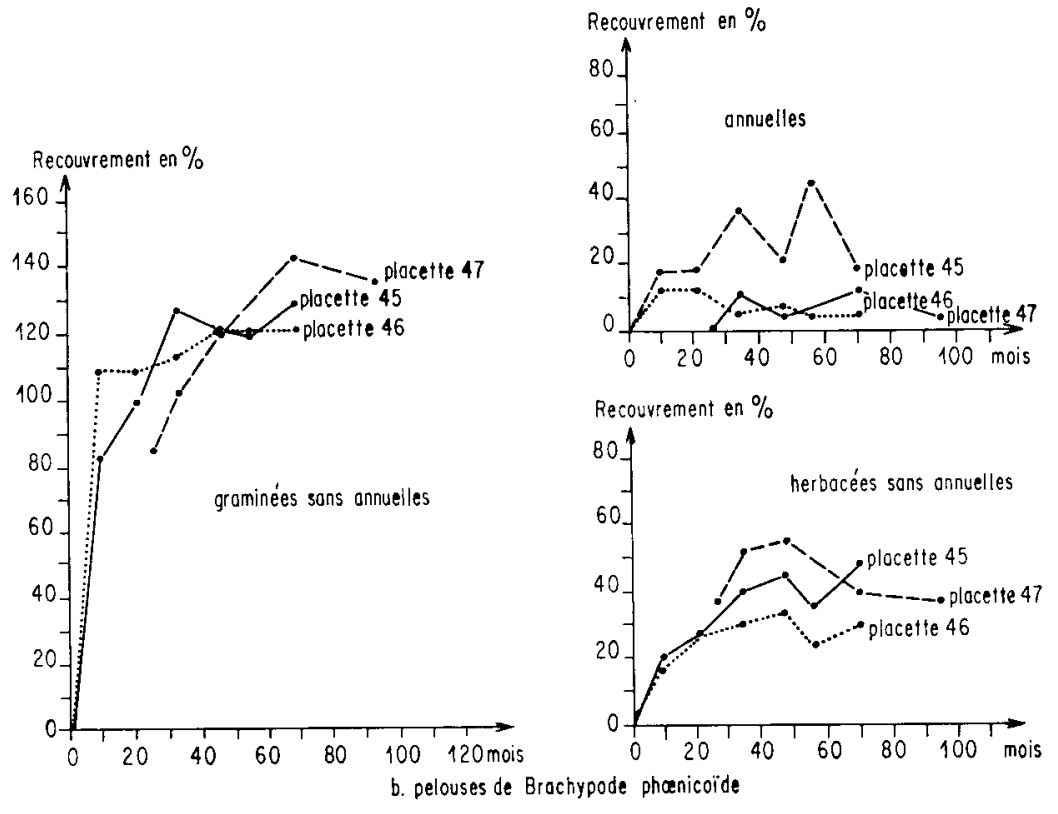

Fig. 6

Evolution, au cours du temps, du recouvrement des végétaux herbacés dans les communautés herbacées incendiées.

Cover changes through time of herbaceous plants in burnt communities initially dominated by herbaceous plants. 


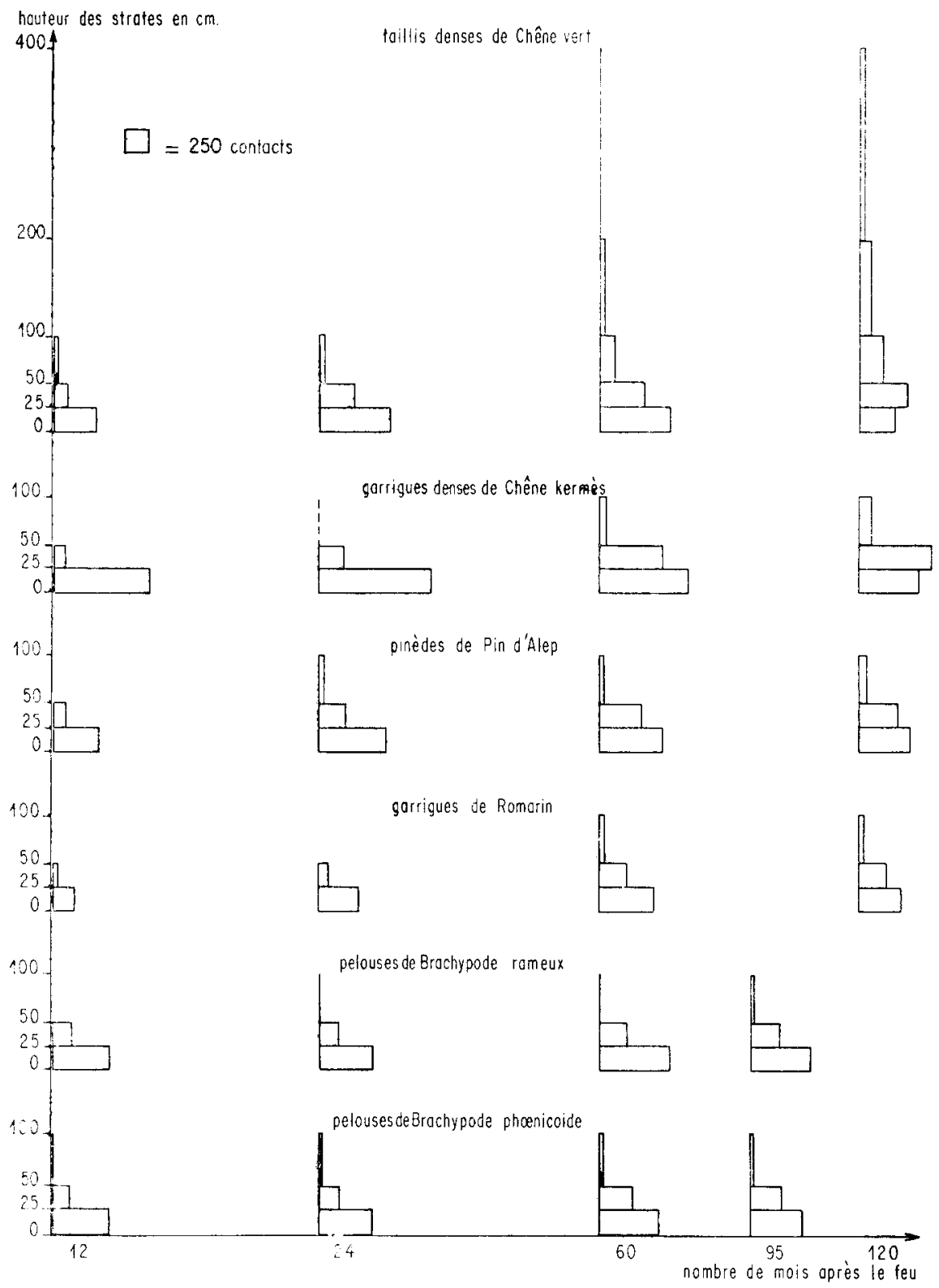

FIG. 7

Evolution, au cours du temps, du nombre de contacts selon les strates, des communautés incendiées.

Changes in the burnt communities of the number of plant contacts according to strata and time. 
Les strates retenues sont les suivantes:

Strate 1: $0-25 \mathrm{~cm}$.
Strate 2: $25-50 \mathrm{~cm}$.
Strate 3: $50-100 \mathrm{~cm}$.
Strate $4: 100-200 \mathrm{~cm}$.
Strate 5: $200-400 \mathrm{~cm}$.

Dans toutes les communautés étudiées, au fur et à mesure qu’elles vieillissent, il y a tendance à l'apparition et à l'augmentation du matériel végétal vers les niveaux élevés tandis que ce matériel tend à diminuer dans les niveaux bas.

Dans les formations de ligneux hauts, le strate 1 apparaît dès les premiers mois suivant le feu (fig. 7); le nombre de contacts atteint son point maximal entre la deuxième et la cinquième année après l'incendie, puis décroît progressivement. Le strate 2 ne présente son point maximal du nombre de contacts que vers la cinquième année, puis reste constante. Seuls les taillis denses de Quercus ilex montreraient une tendance à une légère diminution du nombre de contacts de cette strate, après cette date. La strate 3 , comme la strate 4 , continue sa croissance et ne semble pas avoir atteint le point maximal du nombre de contacts au cours de la période étudiée. La strate 3 n'apparaît réellement qu'à la fin de la première année après le feu, tandis que la strate 4 n'apparaît que vers la troisième année. Pour les taillis denses ou clairs de Quercus ilex, la strate 5 n'apparaît qu'au cours de la sixième année après l'incendie. En un an elle se développe rapidement dans les taillis denses de Quercus ilex, tandis que son développement est plus lent dans les taillis clairs. A la fin de la période des observations, les strates 4 et 5 n'ont pas atteint le point maximal du nombre de contacts. Dans les pinèdes de pin d'Alep les strates 4 et 5 n'apparaissent pas au cours de la période étudiée.

En ce qui concerne les formations dominées par des ligneux bas, les changements de structure sont différents. La strate 1, ainsi que la strate 2, apparaissent dès les premiers mois après l'incendie; mais tandis que la strate 1 atteint son point maximal du nombre de contacts pendant les einq premières années (fig. 7), puis décroît progressivement ( $\mathrm{y}$ compris pour les garrigues de romarin, chez lesquelles cependant le phénomène est moins net), le nombre de contacts de la strate 2 continue à croître et scmble atteindre son point maximal vers la neuvième année après le feu. La strate 3 , en revanche, n’apparaît que vers le milieu de la troisième année. A la fin de la période des observations, le nombre de contacts continuerait à augmenter encore.

Les formations dominces par les végétaux herbacés présentent un développement de la structure différent. Les trois strates, sauf pour les pelouses de brachypode rameux, apparaissent dès la première année après le feu (fig. 7) : les hampes florales et les longues feuilles de Brachypodium phoenicoides atteignent leur taille normale dans l'année, tandis que Brachypodium retusum, espèce présentant une ramification sympodiale à feuilles courtes, mettra plus de temps pour atteindre $50 \mathrm{~cm}$ de hauteur. Dans les pelouses de brachypode rameux, la strate 1 , à la fin de la période des observations, semble ne pas avoir atteint le point maximal de croissance, tandis que chez les pelouses de brachypode phoenicoïde ce stade commence dès la quatrième annéc. Dans ce cas, le fait que Brachypodium phoenicoides soit une graminée présentant de longues tiges florifères et de longues feuilles, entraînerait un phénomène de diminution de la lumière dans la strate 1 au fur et à mesure qu'il se développe, provoquant une disparition, ou une diminution, du nombre d'espèces ou de leur phytomasse à ce niveau. La strate 2 des deux types de pelouses atteint son point maximal au début de la troisième année après le feu, puis demeure constante. Le nombre de contacts de la strate 3 des pelouses 
de brachypode phoenicoïde ne semble plus progresser dès la fin de la troisième année; par contre, pour les pelouses de brachypode rameux, le nombre de contacts de cette strate augmente toujours progressivement. Cela tiendrait aux faits déjà cités : la structure de ramification intrinsèque des deux brachypodes; l'un, Brachypodium phoenicoides émettant des tiges et des feuilles très longues au niveau du sol (comportement d'hémicryptophyte) atteint rapidement son port normal ; l'autre, Brachypodium retusum, émet des tiges et des feuilles au niveau du sol, mais aussi, au fur et à mesure de sa croissance, sur des tiges anciennes (caractère de chaméphyte), il n'atteint donc pas sa taille maximale immédiatement ; dans les taillis de chêne vert, par exemple, à Y'inverse de Brachypodium phoenicoides, Brachypodium retusum peut prendre un aspect lianiforme et atteindre plus d'un mètre de haut.

\section{Discussion et conclusion}

Ainsi, assez rapidement après un incendie, la végétation réapparaît et recouvre la surface du sol; puis progressivement elle deviendra de plus en plus dense. Les communautés étudiées présentent des types d'accroissement horizontal (recouvrement) différents selon qu'elles sont dominées par des végétaux ligneux ou des végétaux herbacés, et cela est dû à la structure et à la composition floristique des communautés avant le feu.

Dans les communautés où les végétaux dominants étaient des ligneux hauts, le recouvrement des phanérophytes ligneux s'accroit régulièrement au fur et à mesure que les communautés avancent en âge. En revanche, le recouvrement des chaméphytes ligneux passe par un maximum puis décroît. Les végétaux herbacés suivent aussi cette évolution. Dans les formations dominćes par des ligneux bas, le recouvrement des phanérophytes ligneux, après un accroissement rapide, tend à rester constant; il en est de même pour les végétaux herbacés; tandis que le recouvrement des chaméphytes ligneux atteint un maximum puis décroît. Dans les communautés dominées par les végétaux herbacés, les phanérophytes ligneux ne jouent qu'un rôle négligeable et leur recouvrement est faible. Par contre, le recouvrement des graminées devient de plus en plus important au fur et à mesure que ces communautés «vieillissent ».

En fait, pour la majorité des communautés dominées par des végétaux ligneux, au fur et à mesure que ceux-ci se développent ct occupent un espace de plus en plus important, le recouvrement de l'ensemble des végétaux herbacés tend à diminuer. Il y a une sorte de compétition pour l'espace entre les différentes formes biologiques, les végétaux ligneux tendant à l'emporter. Cette prédominance des ligneux est affirmée, en outre, très tôt après le feu. Cependant, vers la fin de la période des observations (12 ans environ), un état d'équilibre relatif tend à s'installer : la proportion des diverses formes reste à peu près constante. Cet état est très voisin, sinon déjà identique, de celui des communautés similaires qui n'ont pas été brûlées et sont donc plus âgées.

Les changements de recouvrement des différentes formes biologiques qui apparaissent au cours du temps dénotent la concurrence qui existe entre les végétaux pour occuper la surface du sol, avant que s'établisse un équilibre métastable semblable à celui des communautés plus mûres; cette «succession» des formes biologiques ne fait que projeter horizontalement les changements qui vont survenir au cours du temps dans la structure verticale. 
Tout de suite après le feu apparaît la strate 1 qui est prépondérante pendant les cinq premières années. Ce phénomène est dû à l'abondance de végétaux graminéens, herbacés, et ligncux bas, dominants pendant ces premières années, ainsi qu'à la reprise rapide de la végétation après le feu. Cette croissance serait, d'après certains auteurs (Ahlgren, 1960, 1974; BisWEll, 1974; HARPER, 1977), favorisée par l'apport important de substances nutritives contenues dans les cendres. Progressivement, l'importance de cette strate diminue. Cela est particulièrement net pour les communautés dominées par des végćtaux herbacés. Les strates apparaîtront d'autant plus tardivement qu’elles sont plus élevées.

Au cours des douze ans d'observations, seuls les taillis de chêne vert atteignent la cinquième strate (entre 2 et $4 \mathrm{~m}$ ) (fig. 7). Les pinèdes, bien qu'elles appartiennent aux formations «forestières» ne dépassent pas, généralement, la strate 3 (soit $1 \mathrm{~m}$ ). Cette différence est due au type de stratégie de survie après le feu utilisé par l'espèce principale de chaque communauté : le chêne vert présente de multiples rejets de souche quelques mois après lincendie et atteint très rapidement des tailles importantes (nous avons mesuré, par exemple, $2 \mathrm{~m}$ en 70 mois); tandis que le pin d'Alep ne pouvant se régénérer que par graines n'apparaîtra que l'année suivante et présente une croissance plus lente pendant les premières années après le feu (il atteint, par exemple, $80 \mathrm{~cm}$ en 70 mois).

Dans les communautés considérées comme «fermées », c'est-à-dire où la végétation est très dense (ce sont les taillis de chêne vert et les garrigues de chêne kermès), la strate 1, dix ans après l'incendie, présente un nombre de contacts toujours inférieur à celui de la strate 2 . En fait, la masse végétale dans ces communautés est maximale au niveau de la strate 2. Cela n'est pas le cas pour les autres communautés qui possèdent une végétation plus « ouverte».

Cette situation provient du fait que lorsque les communautés sont jeunes et peu hautes, ce sont les végétaux herbacés et les premiers rejets ligneux proches du sol qui constituent la plus grande partie du nombre des contacts. Par la suite, lorsque les communautés dominées par les végétaux ligneux, qui rejettent de souche, vieillissent, les rameaux inférieurs se lignifient, perdent leurs feuilles, tandis qu'ils émettent des tiges plus jeunes couvertes de nouvelles feuilles. Le maximum de feuilles est toujours situé à la périphérie des végétaux, à l'extrémité des rameaux, en vue d'une utilisation maximale de la lumière. Par conséquent, les strates basses ne sont plus constituées que par des troncs qui offrent moins de contacts. Ce qui n'est pas le cas, ou à un degré moindre, des communautés comme les pinèdes, les garrigues de romarin ou les pelouses de brachypodes, où la lumière peut pénétrer plus facilement à l'intérieur des peuplements du fait de l'architecture même des végétaux qui composent ces peuplements. Ce phénomène a été constaté aussi par Debussche (1978) dans les landes de Cytisus purgans du massif de l'Aigoual après incendie.

Avec le temps, le nombre de contacts des strates élevées des communautés dominées par des végétaux ligneux continuera à croître. Les strates hautes des formations forestières adultes, tels les taillis de chêne vert ou les pinèdes, possèdent le nombre de contacts le plus grand. Ce phénomène est identique dans les vieilles garrigues de chêne kermès (25 ans et plus) (Trabaud, 1980). Dans les communautés dominées par des herbacés, cela ne peut pas se produire, puisque dans nos régions, seules les hampes florifères des végétaux herbacés atteignent ou dépassent $50 \mathrm{~cm}$ de hauteur. A moins qu'il n'y ait un changement de communautés allant vers une succession progressive. 
Ces nouvelles communautés, tendant alors vers des formations ligneuses, seraient forcément différentes des communautés d'origine.

La « remontée du couvert» des strates basses vers les strates hautes et l'apparition d'une multiplicité des strates sont très nettes (fig. 7), y compris pour les pelouses de brachypode rameux, chez lesquelles, pourtant, le phénomène est le plus difficilement décelable. Cette remontée, déjà visible en considérant le nombre absolu de contacts par strate, peut être précisée en utilisant le nombre relatif de contacts de la strate 1 par rapport à l'ensemble des strates de la communauté considérée ; ainsi, par exemple : un an après le feu dans les taillis denses de chêne vert, la strate 1 possède 69,5 p. 100 du nombre total des contacts observés, dix ans après un incendie, cette strate ne représente plus que 16,2 p. 100 du nombre total des contacts de la communauté. En ce quı concerne les pelouses de brachypode rameux, ce phénomène est moins accusé mais varie dans ce sens, ainsi, un an après le feu, le nombre de contacts de la strate 1 représente 76,9 p. 100 ; hüit ans après le feu, il n'en représente que 65,9 p. 100.

Ainsi donc, après un incendie, il y a un retour (une cicatrisation, Godron \& PorsSONET, 1972) plus ou moins rapide selon les communautés, vers un état structural plus complexe d'équilibre métastable, semblable à celui qui existait avant l'incendie. Les communautés non «forestières» atteignent rapidement cet état d'équilibre : environ 2 ans pour les pelouses, et 5 à 6 ans pour les garrigues. En revanche, ce laps de temps, sera beaucoup plus long pour les communautés qui sont dominées initialement par des ligneux hauts : bien plus long que la période des observations.

Les résultats obtenus lors de l'étude sur les changements de la flore (TRABaud \& LEPART, 1980) mettent en évidence que l'évolution de la végétation après le feu suit un modèle appelé par EGLER (1954), modèle de la «composition floristique initiale », c'est-à-dire que toutes les espèces sont présentes immédiatement sur les placettes après le feu, même si par la suite, l'abondance relative des individus change quelque peu. Ce sont les végétaux qui apparaissent tout de suite après le feu, et qui existaient déjà avant le feu, qui se maintiennent. Cela est dû aux types de régénération qu'ils utilisent : soit principalement des organes végétatifs de survie, soit des semences résiduelles enfouies dans le sol. En ce sens, les résultats obtenus peuvent être rapprochés de ceux constatés par les chercheurs américains sur le chaparral californien (végétation similaire à celle de la région des garrigues) lequel se succède à lui-même (SAMPSON, 1944; Horton \& KraEbel, 1955 ; HANES, 1971).

L'accroissement progressif de la phytomasse entraîne une modification de la proportion du matériel végétal des strates basses vers les strates hautes. De même, l'importance relative des formes biologiques (ligneuses et herbacées) change progressivement au cours de l'évolution. En cela, nos observations rejoignent le modèle théorique proposé par LyON (1969, 1971) et ODUm (1971) de simulation de changement de phytomasse apparaissant au cours de la succession après incendie permettant d'expliquer le développement des formes de végétaux au cours du temps.

Reçu pour publication le 30 juin 1982.

\section{Remerciements}

Je remercie particulièrement MM. G. LONG et J. LEPART pour avoir bien voulu revoir et commenter le texte; leurs conseils et critiques m'ont été précieux lors de la rédaction finale. 


\title{
Summary
}

\author{
Post-fire development of the structure of some mediterranean phytocenoses \\ in southern France
}

The post-fire development of the vegetation structure of some phytocenoses in the garrigue area was studied in 47 plots located in Bas-Languedoc (southern France). The direct (or diachronic) method was utilized during the twelve year study of the burnt plots. Eight plant community types representative of the area, from Quercus ilex woodlands to Brachypodium swards, were studied.

After fire the quantitative growth of the vegetation tends to turn back towards a structure similar to that which existed before. Rapidly enough, vegetation reappears and covers the soil surface. Vertical growth was also considered : as communities progressively get older, the importance of the lower layers $(<25 \mathrm{~cm})$ decreases, whereas that of the higher layers increases.

\section{Références bibliographiques}

Ahlgren C.E., 1960. Some effects of fire on reproduction and growth of vegetation in northeastern Minnesota. Ecology, 41, 431-445.

AhlgRen C.E., 1974. Effects of fire on temperate forests : north central United States. In : Fire and ecosystems, T.T. Kozlowski, C.E. Ahlgren eds., Academic Press, New York, 195-223.

Barry J.P., 1960. Contribution à l'étude de la végétation de la région de Nîmes. Année biol., 36, 311-550.

Bharucha F.R., 1932. Etude écologique et phytosociologique de l'association à Brachypodium ramosum et Phlomis lychnitis. Beihefte Bot. Centralb., 2, 247-379.

Biswell H.H., 1974. Effects of fire on chaparral. In : Fire and ecosystems, T.T. Kozlowski, C.E. Ahlgren eds., Academic Press, New York, 321-364.

Braun-Blanquet J., 1936. La forêt d'Yeuse languedocienne (Quercion ilicis). Monographie phytosociologique. Mem. Soc. Etud. Sci. nat. Nîmes, 5, 147 p.

Daget P., Poissonet J., 1971. Une méthode d'analyse phytologique des prairies. Ann. agron., 22, 5-41.

Daget P., Poissonet J., 1974. Quelques résultats sur les méthodes d'étude phytoécologiques, la structure, la dynamique et la typologie des prairies permanentes. Fourrages, 59, 71-81

Debussche M., 1978. Etude de la dynamique de la végétation sur le versant nord-ouest du mont Aigoual. Tome 1, 74 p., tome 2, 67 p., Thèse Doc. Ing. Univ. Sc. Techn. Languedoc, Montpellier.

EgLER F.E., 1954. Vegetation science concept. I. - Initial floristic composition, a factor in old field vegetation development. Vegetatio, 4, 412-417

Godron M., 1968. Quelques applications de la notion de fréquence en écologie végétale. Oecol. Plant., 3, 185-212.

Godron M., Poissonet J., 1972. Quatre thèmes complémentaires pour la cartographic de la végétation et du milieu. Bull. Soc. Lang. Géogr., 6, 329-356.

Godron M., Daget Ph., Emberger L., Long G., Le Floc'h E., Poissonet J., Sauvage C., Wacquant J.P., 1968. Code pour le relevé méthodique de la végétation et du milieu. Ed. C.N.R.S., Paris, 292 p.

HANES T.L., 1971. Succession after fire in the chaparral of southern California. Ecol. Monogr., 41, 27-52.

Harper J.L., 1977. Population biology of plants. Academic Press, New York, 892 p. 
Horton J.S., Kraebel C.J., 1955. Development of vegetation after fire in the chamise chaparral of southern California. Ecology, 36, 244-262.

Kornas J., 1958. Succession régressive de la végétation de garrigue sur calcaires compacts dans la montagne de la Gardiole près de Montpellier. Acta. Soc. bot. pol., 27, 563-596.

Kruger F.J., 1977. Ecology of Cape fynbos in relation to fire. Proc. Symp. Environm. Consequences fire and fuel manage, in medit. ecosystems., U.S.D.A. Forest. Serv., Gen. Techn. Rep. WO. 3, Washington, 230-244.

Kuhnholtz-Lordat G., 1938. La terre incendiće. Essai d'agronomie comparée. La Maison Carrée, Nîmes, 361 p.

Kuhnholtz-Lordat G., 1958. L'écran vert. Mem. Mus. natl. Hist. nat., 9, 276 p.

Levy E., Madden E., 1933. The point method of pasture analysis. N.Z. J. Agric., 46, $267-279$.

LoNG G., 1957. La " 3 step method». Description sommaire et possibilités d'utilisation pour l'observation permanente de la végétation. Bull. Serv. Carte Phytogeogr., 2, $35-43$.

LoNG G., 1958. Description d'une méthode linéaire pour l'étude de l'évolution de la végétation. Bull. Serv. Carte phytogeogr., 3, 107-128.

LYON L.J., 1969. Wildlife habitat research and fire in the northern Rockies. Proc. Annu. Tall Timbers Fire Ecol. Conf., 9, 213-227.

LYON L.J., 1971. Vegetal development following prescribed burning of douglas fir in south central Idaho. U.S.D.A. Forest Serv. Res. Paper INT-105, Intermountain Forest and Range Exp. Stn., 30 p.

NAveh Z., 1974. The ecology of fire in Israel. Proc. Annu. Tall Timbers Fire Ecol. Conf., 13, $131-170$.

NAVEH Z., 1975. The evolutionary significance of fire in the mediterranean region. Vegetatio, 29, 199-208.

Odum E.P., 1971. Fundamentals of ecology. W.B. Saunders Co Philadelphic, 574 p.

Pavillard J.. 1935. Eléments die sociologie végétale. Act. Sci. Indlust., 251, Herman et Cie, Paris, $102 \mathrm{p}$.

Poissonet P., Poissonet J., 1969. Essai de comparaison de méthodes d'analyses phytosociologiques et agronomiques des formations herbacées denses permanentes CN.R.S.C.E.P.E., Montpellier, Doc. 50, 120 p.

Purdie R.W., Slatyiz R.O., 1976. Vegetation succession after fire in sclerophyll woodland communities in southeastern Australia. Aust. J. Ecol., 1, 223-236.

Raunkiaer C., 1905. Types biologiques pour la géographie botanique. Kgl. Danske Videnskab. Selskalss Forhandl., 5, 347-437.

SAMPSON A.W., 1944. PIant succession on burned chaparral lands in northern California. Calif. agric. Exp. Stn, Bull. 685, 144 p.

Soroceanu E., 1936. Recherches phytosociologiques sur les pelouses mésoxérophiles de la plaine languedocienne (Brachypodietum phoenicoidis). Comm. SIGMA, 40, 250 p.

Specht R.L., Rayson P., Jackman M.E., 1958. Dark Island heath (Ninety Miles Plain, South Australia). VI. - Pyric succession : changes in composition, coverage, dry weight and mineral nutrient status. Aust. J. Bot., 6, 59-88.

SWEENEY J.R., 1956. Responses of vegetation to fire. A study of the herbaceous vegetation following chaparral fires. Bot. Publ. Univer. California, 28, 143-250.

SWEENEY J.R., 1967. Ecology of some «fire-type» vegetation in northern California. Proc. Annu. Tall Timbers Fire Ecol. Conf., 7, 110-125.

Trabaud L., 1980. Impact biologique et écologique des feux de végétation sur l'organisation, la structure et l'évolution de la végétation des zones de garrigues du Bas-Languedoc. Thèse Doc. Etat Univ. Sc. Techn. Languedoc, Montpellier, 288 p.

Trabaud L., Lepart J., 1980. Diversity and stability in garrigue ecosystems after fire. Vegetatio, 43, 49-57.

Tutin T.G., Heywood V.H., Burges N.A., Moore D.M., Valentine D.H., Walters S.M., WebB D.A., 1964-1980. Flora europaea. Cambridge Univ. Press, 5 vol., 1 (1964), XXXII + 464 p., 2 (1968), XXVII + 455 p., 3 (1972), XXXIX + 370 p., 4 (1976), XXIX + 505 p., 5 (1980), XXXVI + 452 p. 


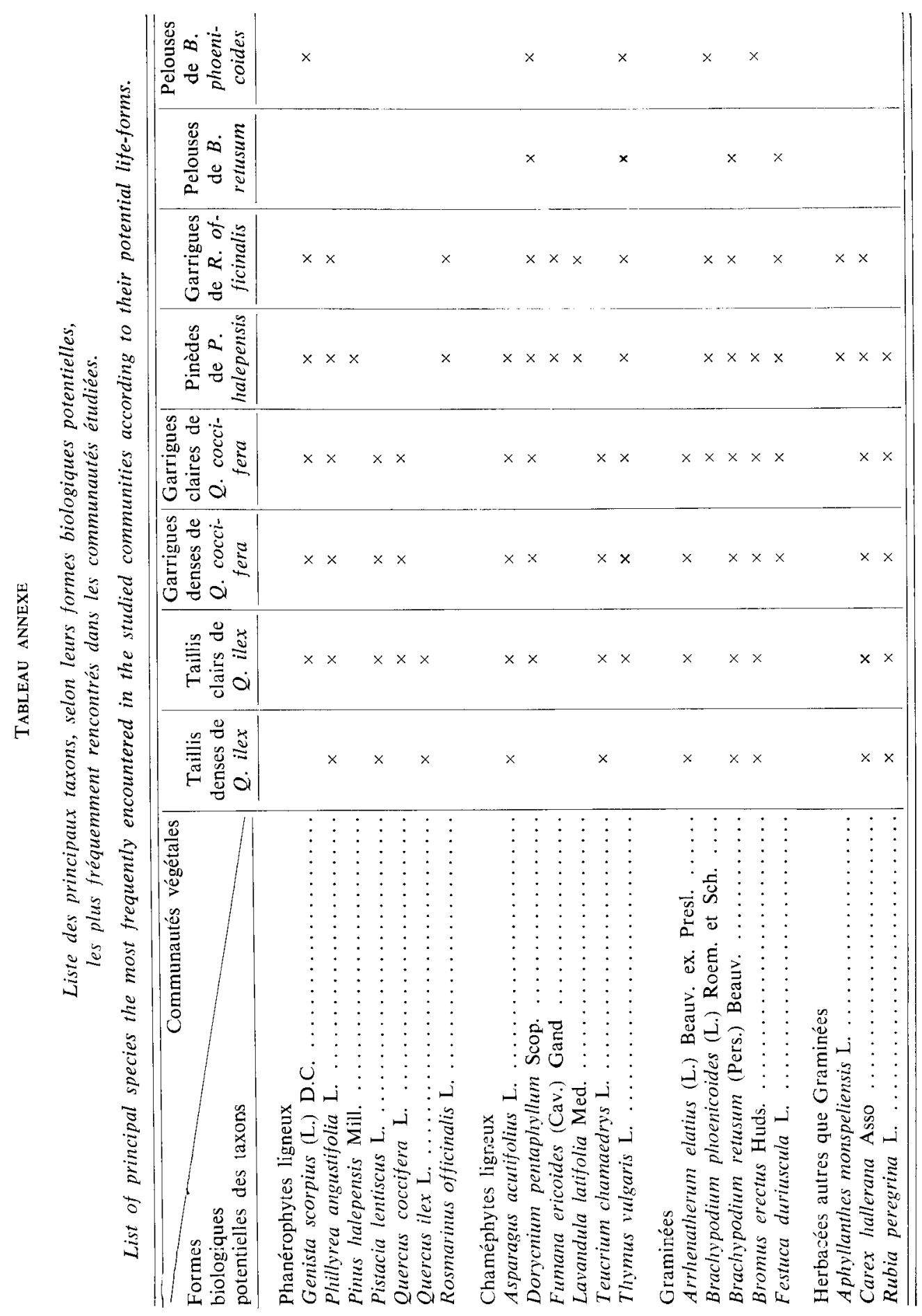

